NASA-CR-204685

\title{
Photoelectron effects on the self-consistent potential in the collisionless polar wind
}

\author{
G. V. Khazanov, M. W. Liemohn, and T. E. Moore \\ Space Sciences Laboratory, NASA Marshall Space Flight Center, Huntsville, Alabama
}

\begin{abstract}
The presence of unthermalized photoelectrons in the sunlit polar cap leads to an enhanced ambipolar potential drop and enhanced upward ion acceleration. Observations in the topside ionosphere have led to the conclusion that large-scale electrostatic potential drops exist above the spacecraft along polar magnetic field lines connected to regions of photoelectron production. A kinetic approach is used for the $\mathrm{O}^{+}, \mathrm{H}^{+}$, and photoelectron $(p)$ distributions, while a fluid approach is used to describe the thermal electrons $(e)$ and self-consistent electric field $\left(E_{\|}\right)$. Thermal electrons are allowed to carry a flux that compensates for photoelectron escape, a critical assumption. Collisional processes are excluded, leading to easier escape of polar wind particles and therefore to the formation of the largest potential drop consistent with this general approach. We compute the steady state electric field enhancement and net potential drop expected in the polar wind due to the presence of photoelectrons as a function of the fractional photoelectron content and the thermal plasma characteristics. For a set of low-altitude boundary conditions typical of the polar wind ionosphere, including $0.1 \%$ photoelectron content, we found a potential drop from 500 $\mathrm{km}$ to $5 R_{E}$ of $6.5 \mathrm{~V}$ and a maximum thermal electron temperature of $8800 \mathrm{~K}$. The reasonable agreement of our results with the observed polar wind suggests that the assumptions of this approach are valid.
\end{abstract}

\section{Introduction}

The polar wind, ion upwelling events, and plasmaspheric refilling are major sources of mass, momentum, and energy for the entire magnetosphere. As the plasma flows up and out of the topside ionosphere, the flow conditions change from subsonic to supersonic, from collision-dominated to collisionless, and from $\mathrm{O}^{+}$dominance to $\mathrm{H}^{+}$dominance. In the collisionless regime, the ion velocity distributions become highly non-Maxwellian, and the coupling between various plasma species occurs through the development of a self-consistent potential.

The reason for the formation of a self-consistent potential in the collisionless plasma is quite clear. High-mobility electrons tend to overtake ions. As a result, the electric neutrality of the plasma is violated and an electric field appears which constrains the electrons, forcing them, on average, to travel together with the ions. This field also significantly affects the motion of the ions by accelerating them. The electric force $e E$ is proportional to the electron temperature $T_{e}$ and has to be combined with the gravitational force $m g$ to determine the pressure distribution of the ions.

Photoelectrons, which form due to ionization of the atmosphere by solar radiation, can alter the self-consistent potential in the space plasma. The presence of the enhanced highvelocity tail in the electron distribution will increase the number of fast ions. Because of enhanced ion acceleration in an expanding plasma, an initial superthermal electron distribution function may be changed.

The polar wind concept was introduced by Banks and Holzer [1968] and Axford [1968]. The latter also suggested that the

This paper is not subject to U. S. copyright. Published in 1997 by the American Geophysical Union.

Paper number $96 \mathrm{JA0} 3343$. lighter ions must be dragged away from the upper atmosphere by the escaping photoelectron flux. Lemaire [1972] pointed out that the additional electrostatic force acting on the ions because of the escaping photoelectrons can accelerate the ions to higher velocities as long as the photoelectron flux is larger than the escape flux of the thermal electrons. This idea was developed further by Barakat and Schunk [1984] by including precipitating hot electrons of magnetospheric origin in the polar cap region. Several tutorials by Schunk [1986, 1988a,b] and the recent review by Ganguli [1996] provide a complete picture of the historical development of polar wind studies.

Recently, motivated by Akebono satellite measurements, Tam et al. [1995a] have developed a numerical steady state polar wind solution that is continuous from the subsonic collisional regime at $500 \mathrm{~km}$ to the supersonic collisionless regime at $2 R_{E}$. Wilson et al. [1996] reexamined this problem in order to reduce the large acceleration of $\mathrm{O}^{+}$ions to supersonic speeds at altitudes below $600 \mathrm{~km}$ and to reduce the high thermal electron temperatures in excess of $40,000 \mathrm{~K}$ at 1000 $\mathrm{km}$ that came from the results of the Tam et al. [1995a] model. They found that by balancing the photoelectron flux with a downward electron flux, instead of an upward $\mathrm{O}^{+}$flux, a potential drop of only 5 to $6 \mathrm{eV}$ results between $500 \mathrm{~km}$ and $3 R_{E}$. They also introduced a precipitating magnetosheath electron flux, or polar rain, to help balance the photoelectron outflow.

The escape of photoelectrons from the sunlit polar cap causes a potential drop to develop that leads to additional ion acceleration. Observations in the topside ionosphere by ISIS 1 [Winningham and Heikkila, 1974] and DE 2 [Winningham and Gurgiolo, 1982] have led these authors to conclude that large-scale electrostatic potential drops exist above the spacecraft along polar magnetic field lines connected to regions of photoelectron production. Pollock et al. [1991] examined the occurrence rate and magnitude of field-aligned electrostatic po- 
tential drops over the ionospheric polar caps. In that study, signatures in upgoing and downgoing photoelectrons were measured in the topside ionosphere using the low-altitude plasma instrument (LAPI) on Dynamics Explorer 2 (DE 2) [Winningham and Gurgiolo, 1982]. These data are compared with ion data obtained at high altitudes using the retarding ion mass spectrometer (RIMS) on the DE 1 spacecraft. Data were selected from intervals when DE 1 and DE 2 were approximately along the same polar cap magnetic field line and when upflowing $\mathrm{O}^{+}$beams were observed in the RIMS data. Pollock et al. [1991] presented one case in which the comparison of data from the two DE spacecraft is quite favorable regarding its interpretations in terms of a field-aligned electrostatic potential drop.

What is the contribution of photoelectrons in the formation of a field-aligned electrostatic potential drop over the polar cap? What is the largest potential drop that should be expected due to the presence of photoelectrons in the polar wind? What is the quantitative relation between the thermal plasma and photoelectron parameters that leads to certain values of the self-consistent electric field in the polar wind? How do the thermal plasma parameters affect the result for a given photoelectron density? These questions are the primary motivation for the study presented in this paper.

\section{General Relations}

In this paper, we will consider the calculation of the upper limit of the potential drop that should be expected in the polar wind due to the presence of photoelectrons. The exclusion of collisional processes leads to easier escape of polar wind particles and therefore to the formation of the largest possible field-aligned electrostatic potential drop over the polar cap. This argument is consistent with the discussion of Tam et al. [1995b] and should only be valid above the region of the source where transport is the dominant process in polar wind formation. In this paper, a kinetic approach is used for the $\mathrm{O}^{+}$, $\mathrm{H}^{+}$, and photoelectron $(p)$ distributions, and a fluid approach is used to describe the thermal electrons $(e)$ and self-consistent electric field $\left(E_{\mathrm{k}}\right)$.

The steady state collisionless kinetic equation can be presented in the following form:

$$
\begin{aligned}
& \mu \mathrm{v} \frac{\partial f_{\alpha}}{\partial s}-\frac{1-\mu^{2}}{2 B} \frac{\partial B}{\partial s} \mathrm{v} \frac{\partial f_{\alpha}}{\partial \mu} \\
& +\left(\frac{e_{\alpha}}{m_{\alpha}} E_{11}-g\right)\left(\mu \frac{\partial f_{\alpha}}{\partial v}+\frac{1-\mu^{2}}{v} \frac{\partial f_{\alpha}}{\partial \mu}\right)=0
\end{aligned}
$$

where $v$ is velocity, $\mu$ is the cosine of pitch angle, $f_{\alpha}=f_{\alpha}(v, \mu, s)$ is the distribution function of species $\alpha, s$ is distance along the geomagnetic field line, $B$ is the geomagnetic field, and $g$ is the gravitational acceleration.

The most delicate part of the polar wind problem in the presence of photoelectrons is photoelectron-thermal electron interactions. In the absence of collisions, the electron plasma component is coupled with $\mathrm{O}^{+}$and $\mathrm{H}^{+}$ions based on the quasineutrality and currentless conditions through the self-consistent electric field. In a steady state collisionless plasma, mass and energy conservation equations for the total electron component can be presented in the form

$$
\frac{\partial}{\partial s}\left(\frac{n u}{B}\right)=0
$$

$$
B \frac{\partial}{\partial s}\left(\frac{Q}{B}\right)-e n E_{||} u=0
$$

where $n, u$, and $Q$ are the moments of electron distribution function

$$
\begin{gathered}
n(s)=\int_{\mathrm{v}} f(\mathrm{v}, \mu, s) d^{3} \mathrm{v} \\
u(s)=\frac{1}{n(s)} \int_{\mathrm{v}} \mu \mathrm{v} f(\mathrm{v}, \mu, s) d^{3} \mathrm{v} \\
Q(s)=\frac{m}{2} \int_{\mathrm{v}} \mu \mathrm{v}^{3} f(\mathrm{v}, \mu, s) d^{3} \mathrm{v}
\end{gathered}
$$

Here $d^{3} v=2 \pi v^{2} d v d \mu$ is the volume in the velocity space. The term with gravitation in (3) can be omitted for electrons.

The electron population in (4) can be separated into two parts: thermal electrons, with distribution function $f_{e}$; and photoelectrons, with distribution function $f_{p}$. Accordingly, the mass and energy conservation equations can be presented as

$$
\begin{gathered}
\frac{\partial}{\partial s}\left(\frac{n_{e} u_{e}+n_{p} u_{p}}{B}\right)=0 \\
B \frac{\partial}{\partial s}\left(\frac{Q_{e}+Q_{p}}{B}\right)-e E_{11}\left(n_{e} u_{e}+n_{p} u_{p}\right)=0
\end{gathered}
$$

Using the kinetic equation (1) for the photoelectron distribution function $(\alpha=p)$, we can find mass and energy conservation for this portion and exclude the photoelectron component from (5) and (6). This leads to the set of thermal electron mass and energy equations

$$
\begin{gathered}
\frac{\partial}{\partial s}\left(\frac{n_{e} u_{e}}{B}\right)=0 \\
B \frac{\partial}{\partial s}\left(\frac{Q_{e}}{B}\right)-e n_{e} E_{\|} u_{e}=0
\end{gathered}
$$

As we pointed out before, these moment equations should be coupled with $\mathrm{O}^{+}, \mathrm{H}^{+}$, and photoelectrons based on the quasineutrality and currentless conditions

$$
\begin{gathered}
n_{e}(s)+n_{p}(s)=n_{\mathrm{O}^{+}}(s)+n_{\mathrm{H}^{+}}(s) \\
n_{e}(s) u_{e}(s)+n_{p}(s) u_{p}(s)=n_{\mathrm{O}^{+}}(s) u_{\mathrm{O}^{+}}(s)+n_{\mathrm{H}^{+}}(s) u_{\mathrm{H}^{+}}(s)
\end{gathered}
$$

where densities and fluxes in (9) for $\mathrm{O}^{+}, \mathrm{H}^{+}$, and photoelectrons must be found based on the solution of the kinetic equation (1).

\section{Solution of the Kinetic Equation}

The solution of the steady state collisionless kinetic equation for polar wind applications has been established and discussed in great detail in several papers by Lemaire and Scherer [1970, 1971, 1972]. They classify the polar wind particles into four categories [Lemaire and Scherer, 1972]: (1) ballistic particles that cannot escape; (2) particles with enough energy to escape to interplanetary space; (3) trapped particles with two mirror points along the field line; and (4) incoming particles from the outermost regions. 
For the purposes of our study, it is assumed that the incoming particles are absent. This assumption leads to an electrostatic potential distribution with no possibility of trapping particles, and so this population is also omitted (see section 7 and Figure 1).

Since the plasma is considered to be collisionless, the timeindependent distribution function in (1) depends only on the particle's total energy

$$
E=\frac{m_{\alpha} v^{2}}{2}+e_{\alpha} U(s)-m_{\alpha} \Psi(s)
$$

and magnetic moment

$$
M=\frac{m_{\alpha} v_{\perp}^{2}}{2 B}
$$

The potential differences $U(s)$ and $\Psi(s)$ can be related to the parallel electric and gravitational fields from the following relations

$$
U(s)=\int_{0}^{s} E_{11}\left(s^{\prime}\right) d s^{\prime} ; \quad \Psi(s)=\int_{0}^{s} g\left(s^{\prime}\right) d s^{\prime}
$$

The particle distribution is constant along any particle trajectory which is characterized by the constants of motion; therefore the distribution function at any point along the geomagnetic field can be calculated provided it is known at some boundary in the ionosphere above the region of the source. For convenience, we will transform our distribution function and integration variables from $(v, \mu)$ to the constants of motion $(E, M)$. The particle density and flux for species $\alpha$ can then be written as

$$
\begin{gathered}
n_{\alpha}=\frac{\pi B}{2}\left(\frac{2}{m_{\alpha}}\right)^{3 / 2} \iint d E d M \frac{f_{\alpha}(M, E)}{\left(E-e_{\alpha} U+m_{\alpha} \Psi-M B\right)^{1 / 2}} \\
n_{\alpha} u_{\alpha}=\frac{\pi B}{2}\left(\frac{2}{m_{\alpha}}\right)^{2} \iint f_{\alpha}(M, E) d E d M
\end{gathered}
$$

which is similar to that found by Whipple [1977] and used by Miller and Khazanov [1993] to calculate a steady state solution for the self-consistent electrostatic potential due to trapped plasma in the magnetosphere.

The particle density at arbitrary positions along the magnetic field can now be easily determined provided the distribution function is known at the lower, ionospheric boundary. The region of determination for the integrals (limits of integration) in (13) is obtained by considering the condition of reflection, which occurs for $v_{\|}=0$, and is given by

$$
v_{\|}=\left(\frac{2}{m_{\alpha}}\right)^{1 / 2}\left(E-e_{\alpha} U+m_{\alpha} \Psi-M B\right)^{1 / 2}=0
$$

which is a function of the total energy of the particle, the local electric and gravitational potentials and magnetic field, and the sign and the mass of the charged particle. Equation (14) depends only on the final position $s^{\prime}$ and is a necessary but not sufficient condition to guarantee accessibility of an ionospheric particle to $s^{\prime}$. For particles to gain access to $s^{\prime}$, they must not be reflected at intermediate positions $\left(0<s<s^{\prime}\right)$ along the magnetic field. According to (14), particles with energy

$$
E \geq e_{\alpha} U\left(s^{\prime}\right)-m_{\alpha} \Psi\left(s^{\prime}\right)+M_{\alpha} B\left(s^{\prime}\right)
$$

reach $s^{\prime}$ and contribute to the particle density $n_{\alpha}\left(s^{\prime}\right)$. Particles having

$$
E<e_{\alpha} U\left(s^{\prime}\right)-m_{\alpha} \Psi\left(s^{\prime}\right)+M_{\alpha} B\left(s^{\prime}\right)
$$

are reflected before reaching $s$. Using these as limits of integration in (13), the particle density and flux for species $\alpha$ can be written as

$$
\begin{aligned}
& n_{\alpha}=\frac{\pi B}{2}\left(\frac{2}{m_{\alpha}}\right)^{3 / 2} \int_{M=0}^{\infty} d M \\
& \times \int_{E=e_{\alpha} U(s)-m_{\alpha} \Psi(s)+M_{\alpha} B(s)}^{\infty} d E \frac{f_{\alpha}(M, E)}{\left(E-e_{\alpha} U+m_{\alpha} \Psi-M B\right)^{1 / 2}} \\
& n_{\alpha} u_{\alpha}=\frac{\pi B}{2}\left(\frac{2}{m_{\alpha}}\right)^{3 / 2} \int_{M=0}^{\infty} \int_{E=e_{\alpha} U(s)-m_{\alpha} \Psi(s)+M_{\alpha} B(s)}^{\infty} f_{\alpha}(M, E) d E d M
\end{aligned}
$$

and depend on the electric and gravitational potentials, magnetic field, and ionospheric distribution function.

\section{Moments of the Velocity Distribution Function}

In order to calculate the hydrodynamic moments (4), the distribution function at the lower boundary of the simulation domain must be specified. For the sake of comparing our results with Tam et al. [1995a], we start at $500 \mathrm{~km}$ (low-altitude boundary) with upper-half Maxwellian ion and photoelectron distributions. It must be noticed, however, that analytical and numerical analyses for the polar wind [Khoyloo et al., 1991] indicate that the adoption of a Maxwellian for an expanding collisionless plasma in the diverging geomagnetic field is inconsistent and causes a discontinuity in the kinetic solutions. Additionally, choosing the lower boundary above the plasma source region introduces additional uncertainties into the problem and predetermines the solution above the lower boundary level (T. I. Gombosi, private communication, 1992).

In our calculations, we separated particles that started from the lower boundary and reached point $s$ on along the field line (part of the escaping population discussed by Lemaire and Scherer [1972]) from particles that have been reflected below this point (a component of the ballistic particles). In accordance with this, we call them the transient and reflected populations. Depending on the electrostatic $e_{\alpha} U(s)$ and gravitational $m_{\alpha} \Psi(s)$ energies of polar wind particles, two cases should be considered separately: case $1, e_{\alpha} U(s)>m_{\alpha} \Psi(s)$, and case 2, $e_{\alpha} U(s)<m_{\alpha} \Psi(s)$.

Case 1 only has a transient population (denoted $t r$ ) and the following expressions for the density and particle fluxes

$$
\begin{gathered}
n_{\alpha}^{t r}(s)=n_{0 \alpha} e^{z_{\alpha}}\left\{1-\Phi\left(z_{\alpha}^{1 / 2}\right)-y \exp \left(\frac{B}{B_{0}} \frac{z_{\alpha}}{y^{2}}\right)\left[1-\Phi\left(\frac{2_{\alpha}^{1 / 2}}{y}\right)\right]\right\} \\
n_{\alpha}^{t r}(s) u_{\alpha}^{t r}(s)=n_{0 \alpha} u_{0 \alpha} \frac{B(s)}{B_{0}}
\end{gathered}
$$

Here subscript " 0 " refers to the lower boundary level; $\Phi$ is the error function,

$$
\Phi(x)=(2 / \sqrt{\pi}) \int_{0}^{x} e^{-t^{2}} d t
$$


and the $z$ and $y$ parameters are defined as

$$
z_{\alpha}=\left|\frac{\left[e_{\alpha} U(s)-m_{\alpha} \Psi(s)\right]}{T_{\alpha}}\right| \quad y=\left[\frac{B_{0}-B(s)}{B_{0}}\right]^{1 / 2}
$$

where $T_{\alpha}$ is the characteristic temperature (energy) of the Maxwellian for species $\alpha$ at the base. The total density and particle flux that should be used in the quasi-neutrality and currentless conditions (9) in this case are

$$
\begin{aligned}
n_{\alpha}(s) & =n_{\alpha}^{\prime r}(s) \\
n_{\alpha}(s) u_{\alpha}(s) & =n_{\alpha}^{t r}(s) u_{\alpha}^{\prime r}(s)
\end{aligned}
$$

Case 2 includes both the transient and reflected (denoted ref) particles, and the following expressions for the density and particle fluxes can be found to be

$$
\begin{gathered}
n_{\alpha}^{t r}(s)=n_{0 \alpha} e^{-z_{\alpha}}\left[1-y \exp \left(-\frac{B}{B_{0}} \frac{z_{\alpha}}{y^{2}}\right)\right] \\
n_{\alpha}^{t r}(s) u_{\alpha}^{t r}(s)=n_{0 \alpha} u_{0 \alpha} e^{-z_{\alpha}}\left[1-y^{2} \exp \left(-\frac{B}{B_{0}} \frac{z_{\alpha}}{y^{2}}\right)\right]
\end{gathered}
$$

and

$$
\begin{gathered}
n_{\alpha}^{r e f}(s)=n_{0 \alpha}\left\{\Phi\left(z_{\alpha}^{1 / 2}\right)-\frac{2}{\sqrt{\pi}}\left(\frac{B_{0}}{B}\right)^{1 / 2} y e^{-z_{\alpha}} F\left[\left(\frac{B}{B_{0}} \frac{z_{\alpha}}{y^{2}}\right)^{1 / 2}\right]\right\} \\
n_{\alpha}^{\text {ref }}(s) u_{\alpha}^{\text {ref }}(s)=n_{0 \alpha} u_{0 \alpha} \frac{B_{0}}{B}\left[y^{2} \exp \left(-\frac{z_{\alpha}}{y^{2}}\right)-e^{-z_{\alpha}}\right]
\end{gathered}
$$

where $F$ is Dawson's integral,

$$
F(x)=e^{-x^{2}} \int_{0}^{x} e^{y^{2}} d y
$$

As was pointed out above, the reflected population in (21) corresponds to the particles that have been reflected below point $s$ back to the base. These quantities are needed at point $s$, though, and the changing cross-sectional area of the flux tube must be taken into account. Therefore the total density and particle flux that should be used in the quasi-neutrality and currentless conditions (9) are

$$
\begin{aligned}
n_{\alpha}(s) & =n_{\alpha}^{\text {tr }}(s)+\frac{B}{B_{0}}\left[n_{\alpha}^{\text {ref }}\left(s^{u b}\right)-n_{\alpha}^{\text {ref }}(s)\right] \\
n_{\alpha}(s) u_{\alpha}(s) & =n_{\alpha}^{\text {tr }}(s) u_{\alpha}^{\text {rr }}(s) \\
& -\frac{B}{B_{0}}\left[n_{\alpha}^{\text {ref }}\left(s^{u b}\right) u_{\alpha}^{r e f}\left(s^{u b}\right)-n_{\alpha}^{r e f}(s) u_{\alpha}^{r e f}(s)\right]
\end{aligned}
$$

Here we add to the transient particles that reached point $s$ the component of particles reflected between point $s$ and the upper level of the simulation domain $s^{\mathrm{ub}}$. The flux equation in (22) can be rewritten in a more simple form as

$$
\begin{aligned}
n_{\alpha}^{t r}(s) u_{\alpha}^{t r}(s) & =n_{0 \alpha} u_{0 \alpha} \frac{B}{B_{s^{\omega b}}} e^{-z_{\alpha}\left(s^{u b}\right)} \\
& \times\left[1-y_{s^{u b}}^{2} \exp \left(-\frac{B_{s^{u b}}}{B_{0}} \frac{z_{\alpha}\left(s^{u b}\right)}{y_{s^{\omega b}}^{2}}\right)\right]
\end{aligned}
$$

where $y_{s}$ ub is taken at the upper boundary level.

\section{Thermal Electron Fluid Equations}

In the previous section, the analytical expressions for the ion and photoelectron moments in the self-consistent coupling with the thermal electron fluid equations (7) and (8) are found as functions of the magnetic and gravitational fields and the self-consistent electrostatic potential. Now we should close the loop and find the corresponding expression for the density of the thermal electrons. The implicit relation for the density of the thermal electrons as a function of the self-consistent electrostatic field can be found from the thermal electron momentum equation

$$
-\frac{\partial\left(n_{e} T_{e}\right)}{\partial s}-\frac{1}{A} \frac{\partial\left(A m_{e} n_{e} u_{e}^{2}\right)}{\partial s}-e n E_{11}=0
$$

where $A$ is the cross-sectional area of the flux tube. Simple integration of this equation leads to the following expression for the thermal electron density

$$
\begin{aligned}
n_{e}(s)= & \frac{1}{T_{e}(s)+m_{e} u_{e}^{2}(s)}\left\{n_{e 0}\left(T_{e 0}+m_{e} u_{e 0}^{2}\right)\right. \\
& \left.-\int_{0}^{s} n_{e}\left(s^{\prime}\right)\left[\frac{\partial U\left(s^{\prime}\right)}{\partial s^{\prime}}+\frac{A^{\prime}}{A} m_{e} u_{e}^{2}\left(s^{\prime}\right)\right] d s^{\prime}\right\}
\end{aligned}
$$

Now, using the condition of quasi-neutrality in (9), an iterative procedure can be used to find the distribution of the selfconsistent potential in the polar wind plasma. Only one parameter in (24) still remains unknown, the electron temperature $T_{e}(s)$. To find it, let us present (8) in the conventional form for the electron temperature by separating the total thermal electron heat flux $Q_{e}$ into the thermal energy flux $Q_{e}^{T}$ and the convective particle motion. Then, using (6) and considering the thermal energy flux as a thermal conductivity flux according to Banks and Kockarts [1973],

$$
Q_{e}^{T}=-\chi_{0} T_{e}^{5 / 2} \frac{\partial T_{e}}{\partial s}
$$

the equation for the thermal electron temperature can be written in the form

$$
\begin{gathered}
B \frac{\partial}{\partial s}\left\{\frac{n_{e} u_{e}}{B}\left(\frac{5}{2} T_{e}+\frac{m_{e} u_{e}^{2}}{2}\right)-\frac{\chi_{0} T_{e}^{5 / 2}}{B} \frac{\partial T_{e}}{\partial s}\right. \\
\left.-e\left(U^{s u b}-U\right) \frac{n_{e} u_{e}}{B}\right\}=0
\end{gathered}
$$

Here $U^{s u b}$ is potential difference at the upper level of the simulation domain, and $\chi_{0}$ is the appropriate constant associated with the thermal conductivity. To calculate the thermal electron particle flux in (26), $n_{e} u_{e}$, the currentless condition in (9) should be used.

Equation (26) can be integrated twice with respect to $s$ and presented as

$$
\begin{aligned}
T_{e}^{7 / 2}= & T_{e 0}^{7 / 2}+\frac{7}{2 \chi_{0}} \int_{0}^{s} B\left\{\frac{n_{e} u_{e}}{B}\left(\frac{5}{2} T_{e}+\frac{m_{e} u_{e}^{2}}{2}\right)\right. \\
& -\left[\frac{n_{e} u_{e}}{B}\left(\frac{5}{2} T_{e}+\frac{m_{e} u_{e}^{2}}{2}\right)\right]_{s u b}+\left[\frac{\chi_{0} T_{e}^{5 / 2}}{B} \frac{\partial T_{e}}{\partial s}\right]_{s \omega b} \\
& \left.-e\left(U^{s^{u b}}-U\right) \frac{n_{e} u_{e}}{B}\right\} d s
\end{aligned}
$$


The square brackets in (27) are taken at the upper boundary of the simulation domain.

It should be pointed out here that in order to provide an analytical description of the electron temperature, we greatly simplified the calculation of the electron thermal flux (25) by using Spitzer's expression for the conductivity coefficient. We will address this issue later in section 7.

Thus our polar wind model uses the kinetic approach to calculate the distributions of photoelectrons and the $\mathrm{O}^{+}$and $\mathrm{H}^{+}$ jons in the polar wind plasma as an explicit analytical function of the self-consistent electrostatic potential (see section 4) and an implicit analytical solution of the fluid equations (24) and (27) to calculate the distribution of the thermal electrons and their temperature, which also contains the self-consistent electrostatic potential. Because of the quasi-neutrality and currentless conditions (9), all polar wind plasma parameters and components are coupled and can be determined based on an iterative scheme.

\section{Iterative Solution Method}

With an initial guess for the $U(s)$ and $T_{e}(s)$ distributions, it is possible to iterate to a convergent solution that satisfies all of the equations. At each iteration step, the quasi-neutrality condition is satisfied using a root-finding method at a given altitude point, and then the electron temperature is calculated based on this new potential and electron density. We start each iteration at the base altitude and work up the field line so the integrals for $n_{e}$ and $T_{e}$ are consistent with the current iteration. The upper boundary terms are taken from the previous iteration, and the process continues until $U(s)$ and $T_{e}(s)$ converge at every step in the spatial domain.

The quasi-neutrality condition in (9) can be rewritten as

$$
F(U)=\sum_{\alpha} e_{\alpha} n_{\alpha}(U)
$$

and then solved for $F(U)=0$. Brent's method was used for this study, which combines the speed of inverse quadratic interpolation with the reliability of the bisection method, and is guaranteed to find the root given an interval that contains one [Press et al., 1992]. After finding an interval of $U$ that contains a root of $F$, this method finds the exact $U$ that balances all of the densities, no matter how rapidly the densities change with $U$.

Since the moments of the kinetic equation only depend on the current spatial step and the upper and lower boundaries, any altitude grid could be used for these equations. However, the numerical integrations in $n_{e}$ and $T_{e}$ dictate that a reasonable step size must be used. This is discussed further in section 7.2 .

One possible problem can arise from the relation between the electrostatic and gravitational potential differences, $e U$ and $m \Psi$. The kinetic formulae above are derived assuming that each plasma species always satisfies either case 1 or case 2. This is not a problem for $\mathrm{H}^{+}$and the photoelectrons, where the particle mass is sufficiently small so $e U$ always dominates. For $\mathrm{O}^{+}$, however, the electrostatic and gravitational potential differences can be similar. For instance, $m_{\mathrm{O}^{+}} \Psi\left(3 R_{E}\right)$ is $7 \mathrm{eV}$, while $e U\left(3 R_{E}\right)$ has been shown to range from $6 \mathrm{eV}$ [Wilson et al., 1996] to $12 \mathrm{eV}$ [Tam et al., 1995a]. Thus it is unknown what case $\mathrm{O}^{+}$will follow, and the potentials could even cross somewhere inside the spatial domain. In general, if $m \Psi$ were to dominate at low altitudes and then be surpassed by $e U$ somewhere up the field line, the governing equations would switch from case 2 to case 1 . In the case 2 equations, the upper boundary would be this crossover altitude, and similarly, in the case 1 equations, this spatial point would be the lower boundary. Should this type of crossover occur in our calculations, the crossover boundary is determined from the previous iteration, but this point is corrected as the calculation proceeds up the field line, if necessary. This could make the lower altitude points inconsistent with the higher altitude solutions, but since the distribution of $U$ is converging with each iteration, these inconsistencies are temporary and will not affect the coherence of the final result. In summary, our algorithm checks for the condition of crossing potentials in the $\mathrm{O}^{+}$calculation and handles it accordingly.

\section{Results}

As we mentioned in the Introduction, the purpose of this study is to answer the following questions: What is the contribution of photoelectrons in the formation of a field-aligned electrostatic potential drop over the polar cap? What is the largest potential drop that should be expected due to the presence of photoelectrons in the polar wind? What is the quantitative relation between the thermal plasma and photoelectron parameters that leads to certain values of the self-consistent electric field in the polar wind? How do the thermal plasma parameters affect the result for a given photoelectron density? These will now be addressed by iteratively solving equations (9), (27), and (28) along a polar cap field line from $500 \mathrm{~km}$ to $5 R_{E}$.

Although there are other factors that affect polar ion motion, such as currents, precipitation, and plasma waves, our objective is to show the impact of just one factor, photoelectrons. Since we are solving the collisionless kinetic equation. we believe we should obtain an upper limit to the photoelectron influence for a given set of input parameters.

The initial guess for $U\left(s^{u b}\right)$ and $T_{e}\left(s^{u b}\right)$ used depends on $n_{p} 0$, the photoelectron content at the base, and ranged from $U\left(s^{u b}\right)$ $=3 \mathrm{~V}$ and $T_{e}\left(s^{u b}\right)=2000 \mathrm{~K}$ for $n_{p 0}=0.0 \%$ up to $U\left(s^{u b}\right)=7 \mathrm{~V}$ and $T_{e}\left(s^{u b}\right)=25000 \mathrm{~K}$ for $n_{p 0}=1.0 \%$. Equations (24) and (27) are sensitive to the $U$ and $T_{e}$ distributions, and so these initial guesses must be reasonable for the solution to converge to a solution. If $U / T_{e}$ is too large, the initial $n_{e}$ distribution will be very low, resulting in large, unrealistic velocities that can cause (27) to have a negative integrand. It was found, however, that the result is independent of the initial guess at $U\left(s^{u b}\right)$ and $T_{e}\left(s^{u b}\right)$, as long as they produce a positive integrand in (27) for the first iteration.

Several initial spatial distributions of these two parameters were also tested. Such choices for $U(s)$ as a linear fit, $U(s)$ proportional to $\left[s /\left(s^{u b}-s_{0}\right)\right]^{1 / 2}$, and $U(s)$ proportional to $\Psi(s)$ produce distributions that converge to the same solution. Likewise for $T_{e}(s)$, a linear fit, $T_{e}(s)$ proportional to $\left[s /\left(s^{u b}\right.\right.$. $\left.\left.s_{0}\right)\right]^{1 / 2}$, and $T_{e}(s)$ proportional to $\sin \left[.5 \pi s /\left(s^{u b}-s_{0}\right)\right]$ all produce distributions that converge to the same solution. Thus the initial spatial distribution of $U$ and $T_{e}$ is irrelevant, as long they produce a positive integrand in (27) for the first iteration.

As follows from the equation for electron temperature (27), we need two boundary conditions to calculate $T_{e}$ : a lower boundary temperature $T_{e O}$ and a temperature gradient at the top of the simulation domain $\left[\partial T_{e} / \partial s\right]_{s} u b$. As we mentioned before, the purpose of this study is to investigate the role of photoelectron escape on the thermal plasma parameters. Because photoelectrons form in the ionosphere and flow out along the 
geomagnetic field lines, their influence on the thermal plasma in the collisionless limit is only through the formation of the self-consistent potential, as seen in (27). To focus on this effect, we will omit magnetospheric energy fluxes by assuming $[\partial T / \partial s]_{s u b=0}$. We will show a case, however, with a source term in the electron temperature equation that accounts for Coulomb collision heating of the thermal electrons by the photoelectrons (see section 7.3).

\subsection{Photoelectron Density Dependence}

To illustrate the photoelectron dependence of our results, typical ionospheric parameters for the polar region were chosen for the low-altitude boundary conditions. The characteristic temperature for each particle species is $2000 \mathrm{~K}$ for the ions, $2500 \mathrm{~K}$ for the thermal electrons, and the photoelectrons have a characteristic energy of $20 \mathrm{eV}$. The $\mathrm{O}^{+}$density at $500 \mathrm{~km}$ is $6 \times 10^{4} \mathrm{~cm}^{-3}$, and $\mathrm{H}^{+}$has $1 \times 10^{3} \mathrm{~cm}^{-3}$, for a total ion density of $n_{\text {tot }}=6.1 \times 10^{4} \mathrm{~cm}^{-3}$. The photoelectron density is varied from $0.00 \%$ to $1.00 \%$ of this number, and the thermal electron density at the base is determined by quasi-neutrality. The density scale is $n_{p 0}=0.01 \%$ to $1.00 \%$ on the Plates because of the logarithmic axis. A photoelectron concentration of $1.00 \%$ at 500 $\mathrm{km}$ is huge and unrealistic but is shown to illustrate an extreme upper limit to the photoelectron influence. Ionospheric polar cap photoelectron densities rarely exceed $0.10 \%$ [Khazanov and Liemohn, 1995].

Although the format of the Plates is unconventional, it is convenient to show the dependence of the results on the photoelectron concentration throughout the polar altitude range.

The electrostatic potential $\varphi$ as a function of the photoelectron relative density at the base and altitude is shown in Plate 1. The potential difference, $U$, is related to the potential by $U(s)=\varphi_{0^{-}} \varphi(s)$, where $\varphi_{0}$ is the potential at $500 \mathrm{~km}$ and $s^{\mu b}$ is a reference altitude where the potential equals zero. For the boundary conditions listed above, $\varphi_{0}$ is $2.87 \mathrm{~V}$ for $n_{p 0}=0.01 \%$, while the total potential for $1.00 \%$ is $7.25 \mathrm{~V}$. It can be seen that the distribution of $\varphi$ is almost constant for $n_{p o}$ above $0.10 \%$, with $\varphi_{0}$ changing less than a volt. The potential also starts to reach an asymptote at low concentrations also. For any $n_{p 0}$. however, most of the potential drop occurs within the first 2 to $3 R_{E}$, smoothing out to zero at the top.

Such electrostatic potential behavior, combined with the gravitational potential, will not create a trap with two mirror points in the spatial domain. Figure 1 shows the total potential energy of the three species that were treated kinetically for a photoelectron concentration of $0.10 \%$ at the base. As you can see, there is no possibility of a "potential well" to trap these particles along the field line. This justifies our assumption earlier that we do not need to include a trapped population in our model.

Plate 2 shows the thermal electron temperature for the same calculation. While $T_{e 0}$ is $2500 \mathrm{~K}$ for all photoelectron densities, the temperature at the top varies from less than $3600 \mathrm{~K}$ at $0.01 \%$ up to $17,300 \mathrm{~K}$ at $1.00 \%$. At any given $n_{p 0}, T_{e}$ rises quickly through the first one to two $R_{E}$, then flattens into a nearly constant temperature for the remainder of the field line. This is due to the thermal conductivity redistributing the energy, smoothing the temperature distribution from the low-altitude source region into the upper altitudes. The energy source for this rise in $T_{e}$ is from the electrostatic coupling of the photoelectrons with the thermal plasma. Notice that the maximum temperature for a realistic photoelectron density $\left(n_{p 0} \leq 0.10 \%\right.$ ) does not exceed $9000 \mathrm{~K}$, in disagreement with the results of Tam et al. [1995a], but consistent with the observations of Abe et al. [1993].

Another interesting result is the ratio of $\mathrm{O}^{+}$to $\mathrm{H}^{+}$densities, shown in Plate 3. The classical polar wind description has $\mathrm{O}^{+}$ dominating at low altitudes and $\mathrm{H}^{+}$dominating at high altitudes. This would appear as a ratio greater than one decreasing to a ratio less than one, reaching unity a few thousand kilometers up the field line. This is exactly what is seen at low $n_{p 0}$ concentrations, with a ratio of unity being reached at less than an $R_{E}$ above the surface and then $\mathrm{H}^{+}$completely dominating the total ion density. As we move up in photoelectron concentration, though, the situation changes as the electrostatic potential grows large enough to drag the $\mathrm{O}^{+}$ions against gravity to higher altitudes. By $n_{p 0}=0.10 \%$, the two ions have similar densities along most of the field line, and, above this photoelectron percentage, $\mathrm{O}^{+}$dominates over the entire spatial region.

The $\mathrm{O}^{+} / \mathrm{H}^{+}$density ratio increases near $5 R_{E}$ at large $n_{p 0}$ concentrations. The ratio at the base is 60 and, for $1.00 \%$ photoelectrons, drops to 28 near $2 R_{E}$ and then increases up to 72 at $5 R_{E}$. This behavior can be explained by examining $z(s)$ for $\mathrm{O}^{+}$, which describes the relationship between the gravitational and electrostatic potential differences. At $1.00 \%, z$ is zero at the base, increases to 4.1 at $2 R_{E}$, and then decreases to 3.65 at the top. Since $\exp (-z)$ is a multiplier in the $\mathrm{O}^{+}$density calculation (see equations (20) and (21)), this increase below 2 $R_{E}$ means that $n_{\mathrm{O}^{+}}$decreases more rapidly than $n_{\mathrm{H}^{+}}$, while the decrease in $z$ above $2 R_{E}$ indicates that $n_{O^{+}}$decreases less rapidly than $n_{\mathrm{H}^{+}}$. These trends in the densities are reflected in the ratios shown in Plate 3.

Another feature that should be discussed is the ion speeds, illustrated here with the Mach number. $M_{\mathrm{O}^{+}}$is given in Plate 4 and $M_{\mathrm{H}^{+}}$is given in Plate 5. For these plots, the Mach number is defined as the bulk velocity at a given altitude divided by the thermal velocity at the base. Therefore all of these Mach numbers are relative to an ion thermal speed for a $2000 \mathrm{~K}$ Maxwellian distribution, that is, $0.81 \mathrm{~km} \mathrm{~s}^{-1}$ for $\mathrm{O}^{+}$and 3.2 $\mathrm{km} \mathrm{s}^{-1}$ for $\mathrm{H}^{+}$.

In Plate $4, M_{\mathrm{O}^{+}}$changes from subsonic for the entire field line at $0.01 \%$ to supersonic for most of the field line at $1.00 \%$. This subsonic region corresponds to the classical polar wind

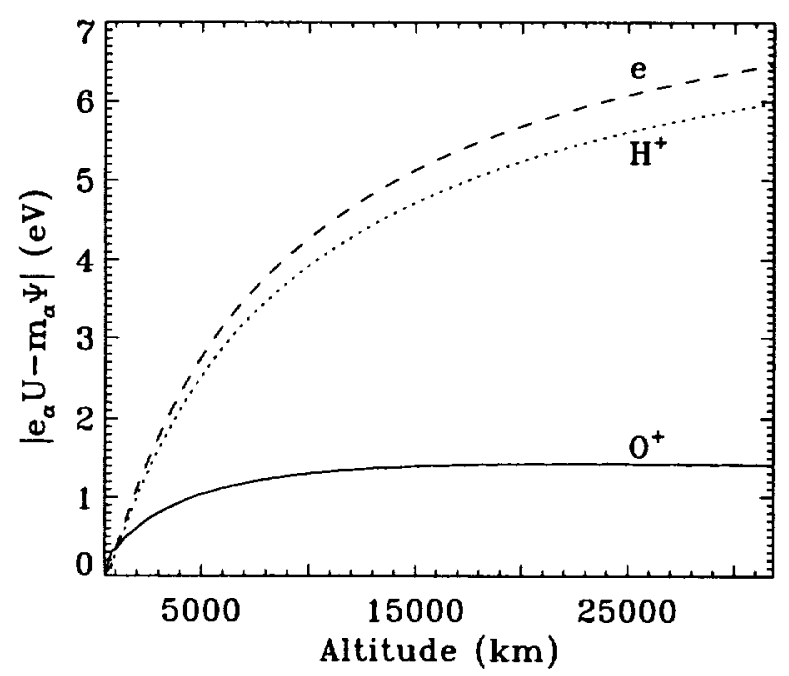

Figure 1. Total potential energy for $\mathrm{O}^{+}, \mathrm{H}^{+}$, and electrons as a function of altitude for $n_{p 0}=0.10 \%$. 


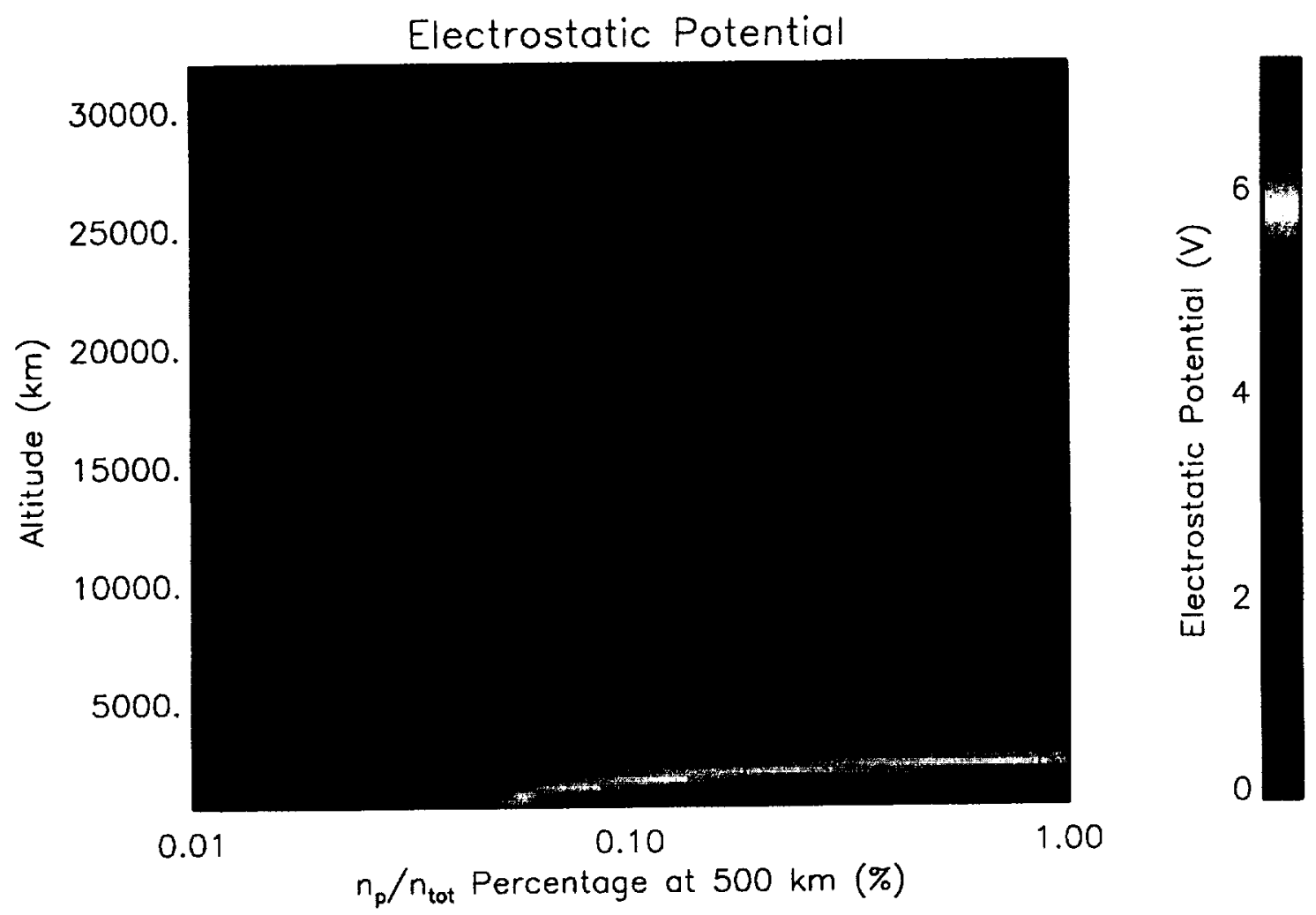

Plate 1. Electrostatic potential as a function of photoelectron density and altitude. Low-altitude boundary conditions are $\mathrm{O}^{+}$density of $6 \times 10^{4} \mathrm{~cm}^{-3}, \mathrm{H}^{+}$density of $1 \times 10^{3} \mathrm{~cm}^{-3}, T_{i 0}=2000 \mathrm{~K}, T_{e 0}=2500 \mathrm{~K}$, and $E_{p 0}=20 \mathrm{eV}$.

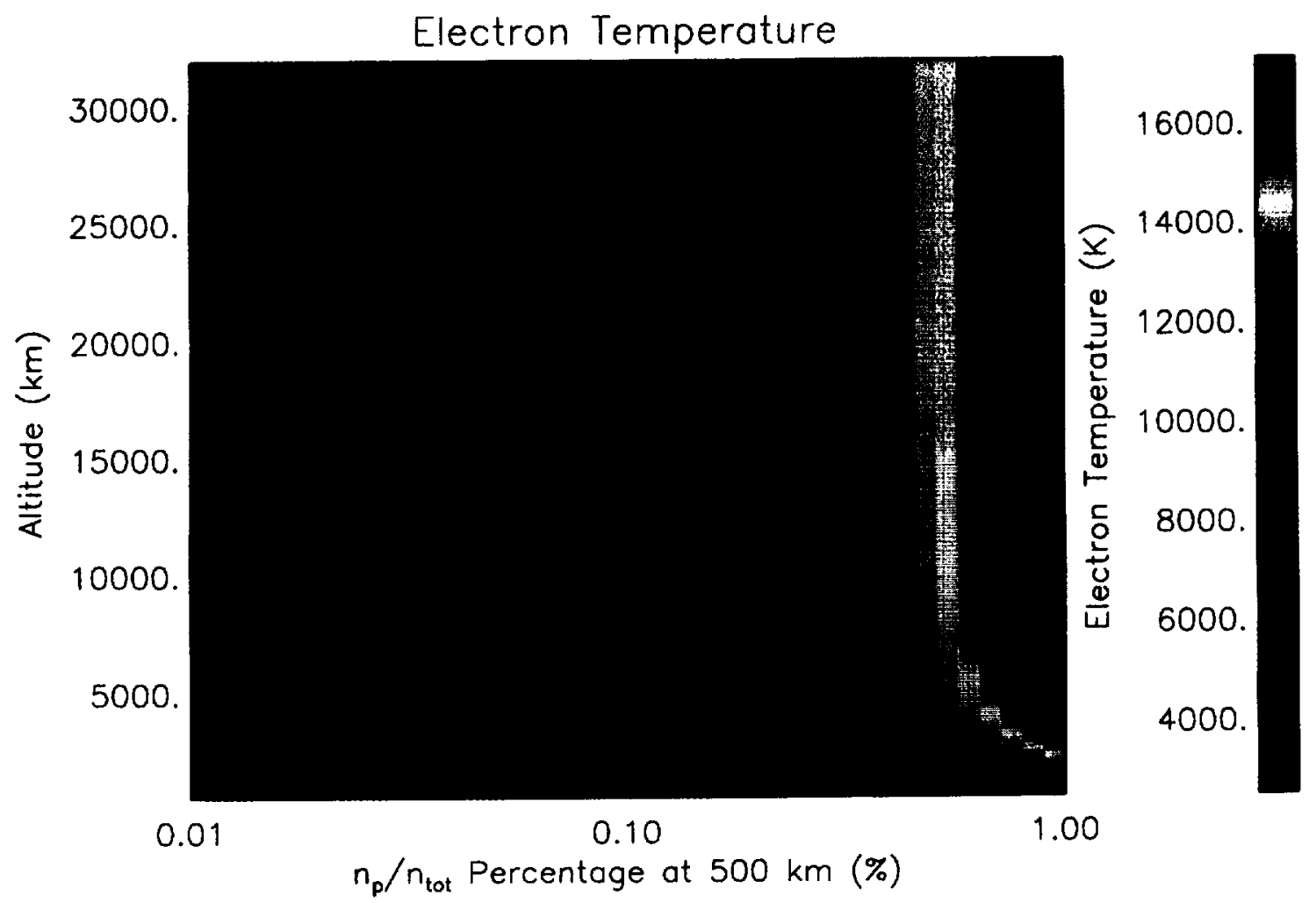

Plate 2. Thermal electron temperature as a function of photoelectron density and altitude. Same boundary conditions as Figure 1. 


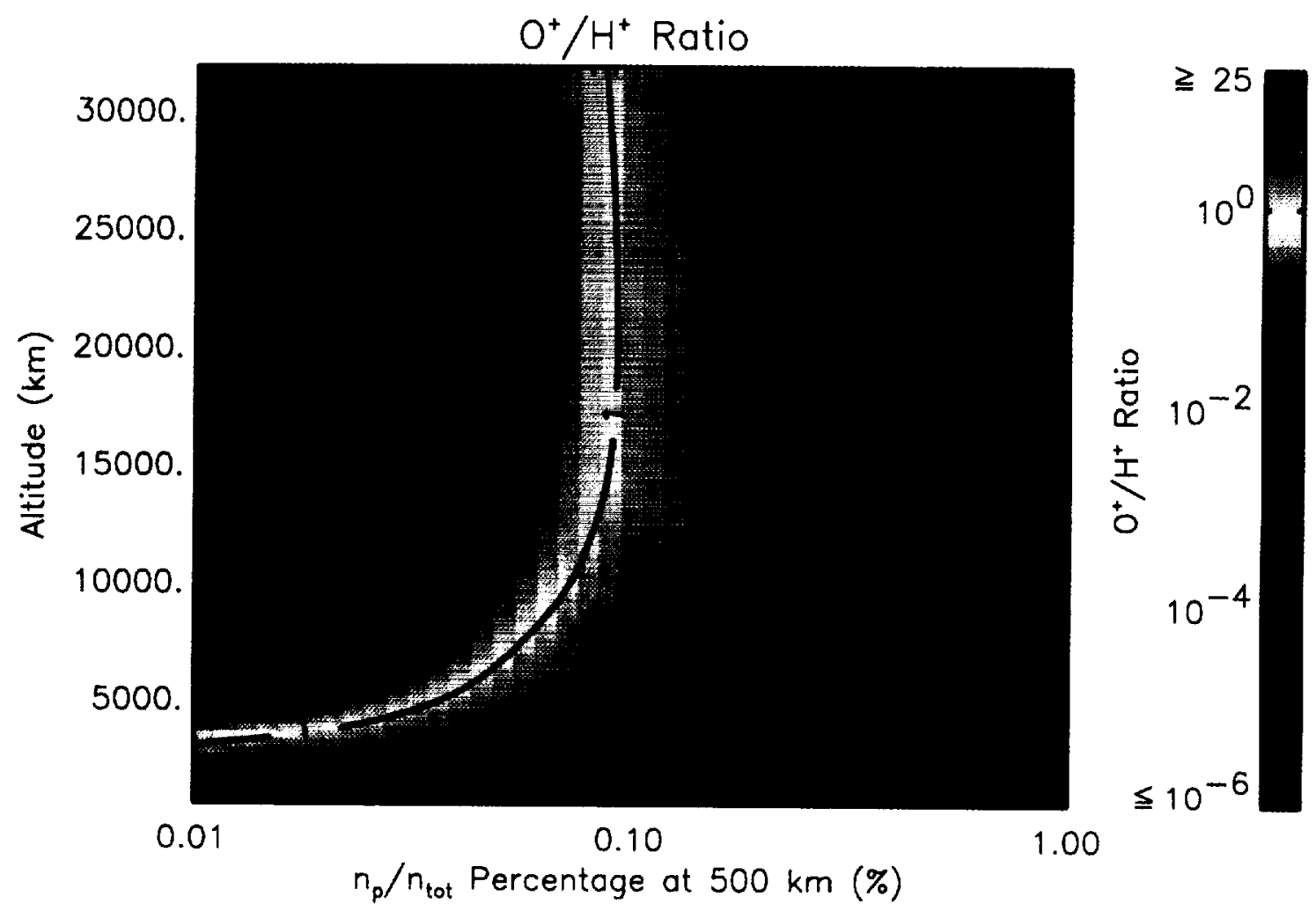

Plate 3. $\mathrm{O}^{+} / \mathrm{H}^{+}$density ratio as a function of photoelectron density and altitude. Same boundary conditions as Figure 1.

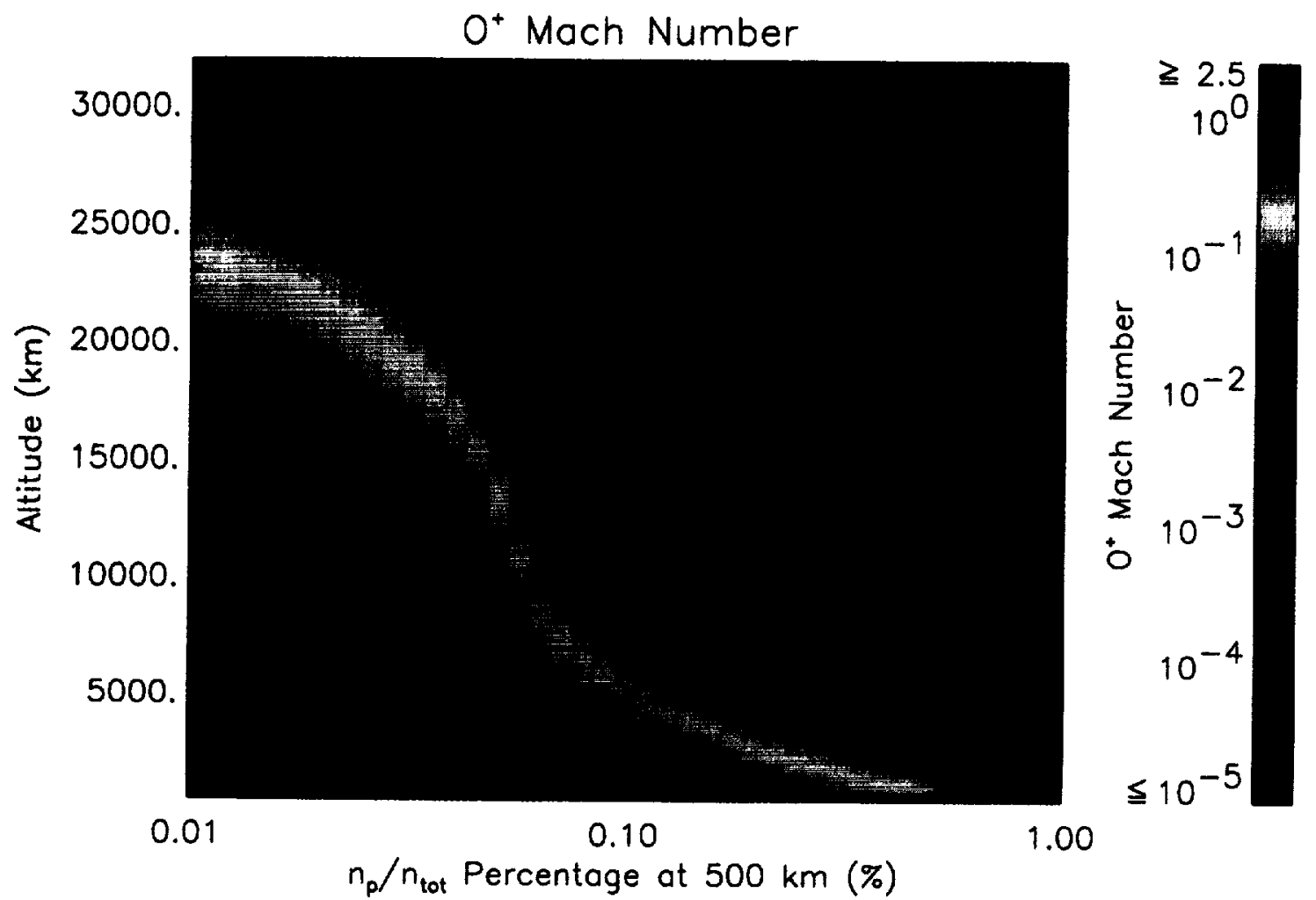

Plate 4. $\mathrm{O}^{+}$Mach number as a function of photoelectron density and altitude. Same boundary conditions as Figure 1. 


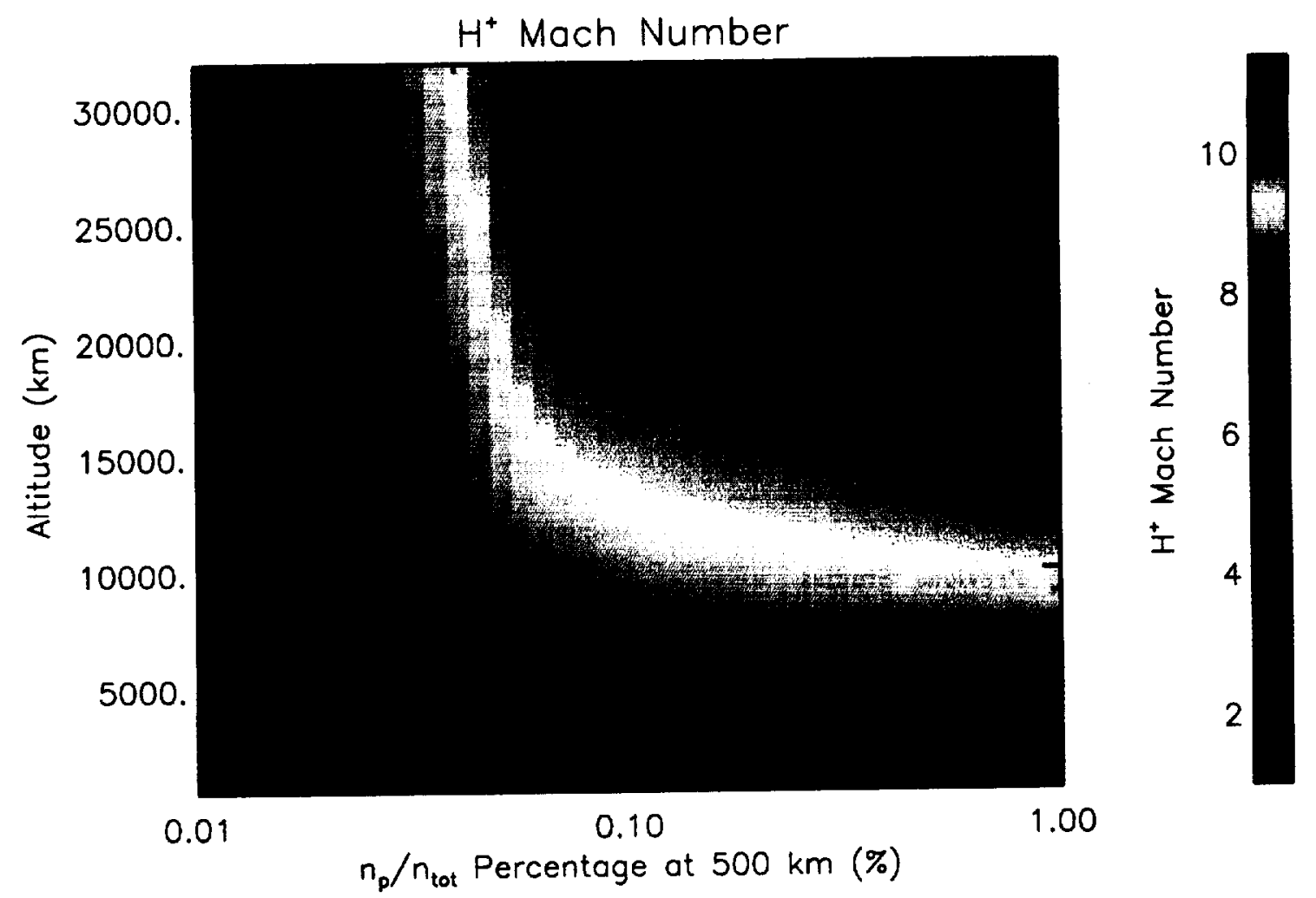

Plate 5. $\mathrm{H}^{+}$Mach number as a function of photoelectron density and altitude. Same boundary conditions as Figure 1.

region discussed with Plate 3 , with velocities orders of magnitude less than the thermal speed for the first few $R_{E}$ up the field line. In the opposite extreme, for example, $n_{p 0}=1.00 \%, \mathrm{O}^{+}$ becomes supersonic by $1500 \mathrm{~km}$, reaches a Mach number of 2.7 near $2 \mathrm{R}_{\mathrm{E}}$, and then slowly lessens to $M_{\mathrm{O}}+\approx 1.1$ at the top. This appears to be an asymptotic limit for the $\mathrm{O}^{+}$Mach number at high altitudes, corresponding to a velocity just under 1 $\mathrm{km} \mathrm{s}^{-1}$. This maximum in the $\mathrm{O}^{+}$velocity near $2 R_{E}$ is again due to the difference in the spatial dependencies of the gravitational and electrostatic potentials.

The protons, however, are always supersonic for the given initial parameters (Plate 5). Mach 5 is achieved between 2000 and $2700 \mathrm{~km}$ altitude after a quick acceleration, and then it seems to increase more slowly above this level. Again, a clear separation between the classical polar wind and a different state is evident. At low $n_{p 0}$ values, the Mach number reaches 7 to 8 at the top, but at higher $n_{p o}$ values, the speeds break Mach $11\left(35 \mathrm{~km} \mathrm{~s}^{-1}\right)$. Even with this difference at higher altitudes, the main acceleration region for $\mathrm{H}^{+}$is at low altitudes, regardless of the photoelectron concentration.

\subsection{Boundary Condition Dependence}

It is also useful to determine the influence of changing various other parameters of the calculation. Increasing the number of spatial points has very little affect on the results. In doubling the number of altitude levels from 100 to $200, T_{e}$ and $\varphi$ are not significantly changed ( $<1 \%$ difference), showing that the numerical integrations are consistent. Changing the upper boundary level also has very little affect on the results. Dropping $s^{u b}$ to $3 R_{E}$ creates less than $5 \%$ difference in $T_{e}$ and less than $1 \%$ difference in $\varphi$.

The choice of characteristic energies at $500 \mathrm{~km}$ can be more significant, but they are still not crucial parameters. Increasing the photoelectron energy to $40 \mathrm{eV}$, or even $60 \mathrm{eV}$, causes only a minor correction to the results, increasing $T_{e}$ by roughly $15 \%$ for each of these steps. This acts to decrease $n_{p}$ and increase $n_{e}$ and $n_{O^{+}}$slightly, and $\mathrm{O}^{+}$becomes supersonic earlier, dropping from $2 R_{E}$ for $E_{p 0}=20 \mathrm{eV}$ to $1.5 R_{E}$ for $E_{p 0}=60 \mathrm{eV}$. The electrostatic potential is not significantly changed. Increasing $T_{e 0}$ to $4000 \mathrm{~K}$ only affects the first few spatial points, and by $1500 \mathrm{~km}$, the influence on $T_{e}(s)$ is less than $1 \%$. The variable $\varphi_{0}$ is $0.1 \mathrm{~V}$ higher, but $\varphi(s)$ drops faster in these initial points, and is then similar throughout the spatial range. This acts to increase the $\mathrm{O}^{+}$velocity near the base, but the velocity is unchanged above $1 R_{E}$. Changing all thermal temperatures at the base to $4000 \mathrm{~K}$ acts to decrease $\varphi$ by $20 \%$ below $2 R_{E}$. At this altitude, $n_{\mathrm{H}^{+}}$has doubled and $n_{\mathrm{O}^{+}}$has tripled over the previous results. There is a similar jump in the velocities, but the Mach numbers are very similar to the previous case. This decrease in the potential is because the ions are already moving faster, therefore less of an assist from the electrons is needed to lift them along the field line.

Another point to discuss is the dependence of the results on the reflected particle population. Removal of these terms from the case 2 equations yields less than a $1 \%$ correction in the electrostatic potential and less than $0.1 \%$ difference in the electron temperature. The spatial distribution of the photoelectron density is affected, however, dropping to almost half of its value of when reflection was included. The $\mathrm{O}^{+}$density distribution is also changed, dropping by an amount matching the photoelectron depletion. There is no significant effect on $\mathrm{H}^{+}$or the thermal electrons. From this, it can be mentioned that the reflected populations play only a very small role in the main results of this paper. 
Table 1. Comparison With Lemaire [1972]

\section{$n_{p 0}, \% \quad$ Lemaire [1972] This study}

\begin{tabular}{llll}
\hline $\mathrm{O}^{+}-\mathrm{H}^{+}$crossover & $\leq 0.0094$ & $5800 \mathrm{~km}$ & $4300 \mathrm{~km}$ \\
$n_{i}$ at crossover & $\leq 0.0094$ & $4.6 \mathrm{~cm}^{-3}$ & $25 \mathrm{~cm}^{-3}$ \\
$n_{\mathrm{H}^{+}}(10,000 \mathrm{~km})$ & $\leq 0.0094$ & $1.7 \mathrm{~cm}^{-3}$ & $5.9 \mathrm{~cm}^{-3}$ \\
$n_{\mathrm{O}^{+}}(10,000 \mathrm{~km})$ & 0.0 & $8.0 \times 10^{-2} \mathrm{~cm}^{-3}$ & $2.7 \times 10^{-2} \mathrm{~cm}^{-3}$ \\
$n_{\mathrm{O}^{+}}(10,000 \mathrm{~km})$ & 0.0094 & $0.14 \mathrm{~cm}^{-3}$ & $2.9 \times 10^{-2} \mathrm{~cm}^{-3}$ \\
$n_{p}(10,000 \mathrm{~km})$ & 0.0094 & $2.2 \times 10^{-2} \mathrm{~cm}^{-3}$ & $3.6 \times 10^{-2} \mathrm{~cm}^{-3}$ \\
$u_{\mathrm{H}^{+}}(h \rightarrow \infty)$ & 0.0094 & $21 \mathrm{~km} \mathrm{~s}^{-1}$ & $21 \mathrm{~km} \mathrm{~s}^{-1}$ \\
\hline
\end{tabular}

\subsection{Comparisons With Previous Studies}

It is useful to compare our polar wind results with other calculations for this region. The first comparison will be with the results of Lemaire [1972], who calculated the steady state polar wind kinetically for all four species $\left(\mathrm{O}^{+}, \mathrm{H}^{+}\right.$, and thermal and superthermal electrons). This model was the first to consider photoelectrons in the polar wind, and predicted modest changes due to the new electron population. The low altitude boundary of Lemaire [1972] was at $950 \mathrm{~km}$, with $7 \times 10^{3} \mathrm{~cm}^{-3}$ for $\mathrm{O}^{+}$and $320 \mathrm{~cm}^{-3}$ for $\mathrm{H}^{+}$, varying the photoelectron content from $0.0 \%$ to $0.0094 \%$. These photoelectron concentrations are below those shown in section 7.1 but are valid compositions since the solar zenith angle is large at polar cap latitudes. The thermal plasma temperature was taken to be $3000 \mathrm{~K}$ everywhere, and the photoelectrons were assumed to have a characteristic energy of $10 \mathrm{eV}$.

Results of this comparison are given in Table 1 . As in the work of Lemaire [1972], our model also shows that $n_{\mathrm{H}^{+}}$is not affected by the presence of photoelectrons at the concentrations taken for these calculations $(0.0 \%$ and $0.0094 \%)$. Also, both models predict a crossover of the $\mathrm{O}^{+}$and $\mathrm{H}^{+}$densities at an altitude of less than an $R_{E}$. It is clear then that these concentrations are very small and the polar wind could still be considered classical. Notice that in Table 1, the first three parameters shown have no significant dependence on the varying photoelectron concentrations used by Lemaire [1972]. Also note that the models predict the same $\mathrm{H}^{+}$flow velocity at $h \rightarrow \infty$ of $21 \mathrm{~km} \mathrm{~s}^{-1}$. The other parameters, although quantitatively different, show the same general trend, including a slight increase in the $\mathrm{O}^{+}$density at higher altitudes but not at the $\mathrm{O}^{+}-\mathrm{H}^{+}$crossover altitude.

Since the recently developed model of Tam et al. [1995a] is also a steady state polar wind solution, this provides a good opportunity for assessment. It must be mentioned that the models are different since Tam et al. [1995a] is a numerical solution that includes collisions. We believe, however, that choosing the low-altitude boundary at $500 \mathrm{~km}$, above the region of the source, is a more critical step than omitting collisional processes in the simulation region, becuase this predetermines the solution above the lower boundary level [T. I. Gombosi, private communication, 1992]. Here we will point out the similarities and differences in the results.

For this comparison, we tried to match the Tam et al. [1995a] boundary conditions as closely as possible. The plasma densities at $500 \mathrm{~km}$ were taken to be $6 \times 10^{4} \mathrm{~cm}^{-3}$ for $\mathrm{O}^{+}, 1 \times 10^{3} \mathrm{~cm}^{-3}$ for $\mathrm{H}^{+}$, and $0.1 \%$ of the balancing electrons for photoelectrons. The characteristic temperatures at the base are $4500 \mathrm{~K}$ for the ions, $5000 \mathrm{~K}$ for the electrons, and $20 \mathrm{eV}$ for the photoelectrons. Our simulation domain extended up to 5 $R_{E}$, but only the results up to $2 R_{E}$ are shown in the comparison, as was presented by Tam et al. [1995a]. As pointed out in section 7.2 , the location of the upper boundary has little effect on the results below $2 R_{E}$.

Figure 2 shows the results from the two models. In each plot, the photoelectron content used to produce each curve is indicated for our results, and the results of Tam et al. [1995a] are indicated with T95 and curves in bold. Similar quantities

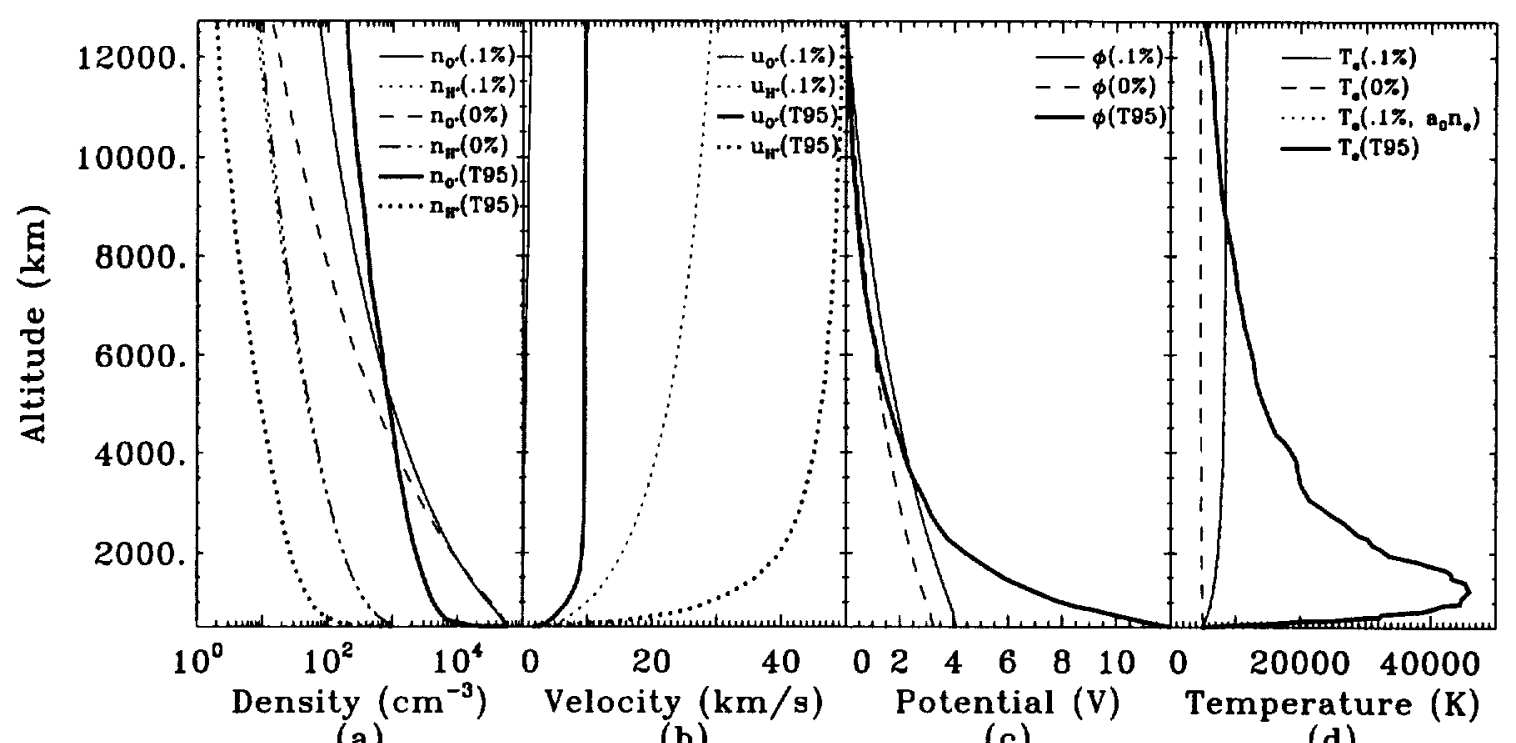

Figure 2. Comparison of results with those of Tam et al. [1995], showing (a) ion density, (b) ion bulk velocity, (c) electrostatic potential, and (d) thermal electron temperature from $500 \mathrm{~km}$ to $2 R_{E}$. Our results are indicated with the base photoelectron density used and theirs are indicated by T95 and bold linestyles. Lowaltitude boundary conditions are $\mathrm{O}^{+}$density of $6 \times 10^{4} \mathrm{~cm}^{-3}, \mathrm{H}^{+}$density of $1 \times 10^{3} \mathrm{~cm}^{-3}, T_{i 0}=4500 \mathrm{~K}, T_{e O}=5000$ $\mathrm{K}$, and $E_{p 0}=20 \mathrm{eV}$. 
are shown with the same linestyle, that is, $\varphi(0.1 \%)$ and $\varphi($ T95) are both solid lines, while $\varphi(0.0 \%)$ is a dashed line.

There is a good deal of agreement in the results of the models. In Figure 2a, it appears that above $2000 \mathrm{~km}$ the $\mathrm{H}^{+}$ densities have the same scale height, and above $4000 \mathrm{~km}$, the $\mathrm{O}^{+}$densities are similar in magnitude. Notice that both models predict that $\mathrm{O}^{+}$is always the dominant ion. It is clear from Figure $2 \mathrm{~b}$ that both models predict a much higher bulk velocity for $\mathrm{H}^{+}$than for $\mathrm{O}^{+}$. The tendencies for each species is also quite similar. Figure $2 \mathrm{c}$ shows that the potentials calculated by the models above $2000 \mathrm{~km}$ are quite close. For this plot, $\varphi\left(2 R_{E}\right)$ is taken to be zero. Also, the thermal electron temperatures are comparable above $6000 \mathrm{~km}$ (Figure 2d).

The difference in the results at low altitudes $(s \leq 2000 \mathrm{~km})$ is clearly seen in the larger potential calculated in the Tam et al. [1995a] model. This is probably related to the very large and unrealistic electron temperatures that the Tam et al. [1995a] model predicts for these altitudes, as compared with Akebono satellite data given by Abe et al. [1993]. The Tam et al. [1995a] model also calculates increased ion acceleration and decreased ion densities in this region.

Our results reproduce the Akebono temperature profiles of Abe et al. [1993] very well in the low-altitude range. Figure 3 shows this comparison. The data is from April 28 and May 10, 1991, as the satellite passed from the dayside to the nightside at progessively higher altitudes. They presumed the data fluctuations were latitudinal or local time variations in the ionospheric conditions. Shown with the data are three curves at different levels of $n_{p o}(0.02 \%, 0.03 \%$, and $0.06 \%)$, which are reasonable numbers for the photoelectron content based on calculations from the Khazanov and Liemohn [1995] model for the conditions of an illuminated polar cap. This comparison shows that this data can be reproduced by this model for realistic estimates of the photoelectron content at the base.

Also of interest in Figure 2 is the comparison of our results with and without photoelectrons. Figure 2a shows that the proton density is unaffected by the presence of photoelectrons, being driven primarily by the changing magnetic field. This conclusion was first mentioned by Lemaire [1972], and was also illustrated earlier in this section. $\mathrm{O}^{+}$, however, shows a definite decrease in density without photoelectrons, and the $\mathrm{O}^{+} / \mathrm{H}^{+}$density ratio is unity just above $2 R_{E}$. From Figure $2 \mathrm{c}$, the photoelectrons appear to increase $\varphi_{0}$ by nearly a volt, but $\varphi(0.1 \%)$ and $\varphi(0.0 \%)$ have comparable spatial distribution trends. Comparing the thermal electron temperature profiles with and without photoelectrons, it is clear that there is an influence. The result without photoelectrons actually decreases with altitude just a bit, while the $T_{e}(0.1 \%)$ nearly doubles between $500 \mathrm{~km}$ and $2 R_{E}$.

One other result plotted here is our model with a source term included in the thermal electron temperature equation. This source term represents energy deposition through Coulomb collisions with the photoelectrons. Although this term is inconsistent with the collisionless nature of the model, it is useful to illustrate the effects of including electron-electron collisions. This source term is expressed through the simple expression $a_{0} n_{e}$ [Khazanov et al., 1992], where $a_{0}$ represents the energy input rate to a single thermal electron from collisions with photoelectrons. Here a value of $5 \times 10^{-4} \mathrm{eV} \mathrm{s}^{-1}$ is used, a typical heat input for a dayside electron at midlatitudes. This quantity is an overestimation of the heating expected in the polar region, since it was determined from a case where two conjugate ionospheres contribute to the photoelectron con-

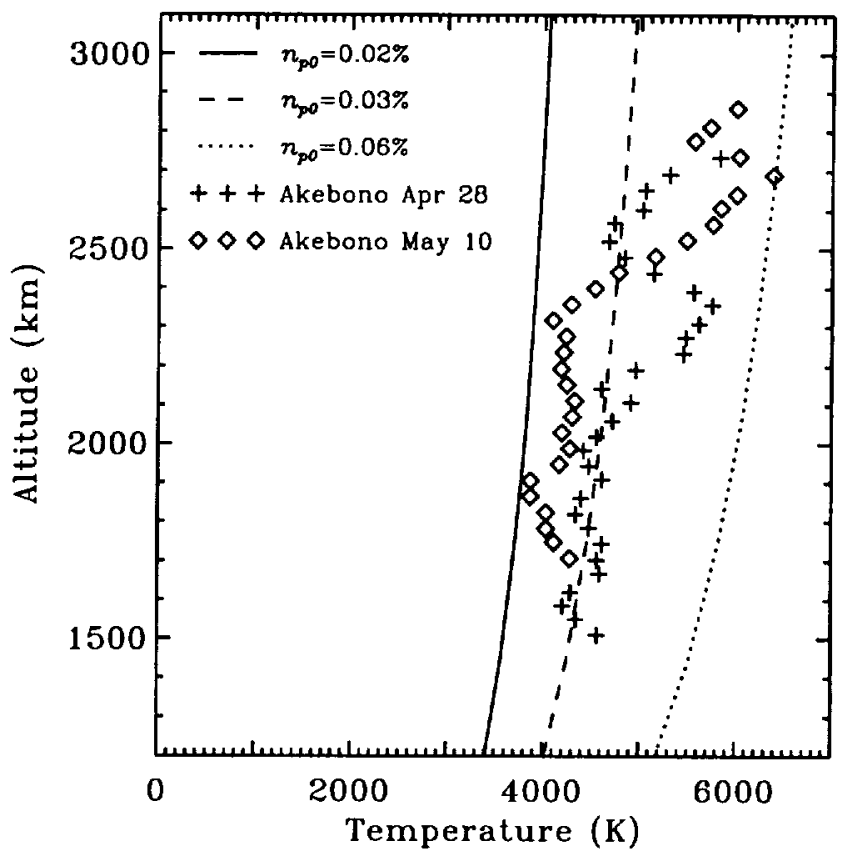

Figure 3. Comparison of results for several values of $n_{p 0}$ with data from Abe et al. [1993] from April 28 and May 10, 1991.

tent. This term replaces the zero on the right hand side of (26), and so contributes to the temperature equation (27) as

$$
\int_{s}^{s^{u b}}\left(a_{0} n_{e} / B\right) d s
$$

inside the integral on the right-hand side. The influence of this term is shown in Figure $2 \mathrm{~d}$, and it is clear that this source is not a significant factor in changing the thermal electron temperature in the polar wind, raising it by less than $1 \%$ throughout the altitude range.

The issue of the calculation of the electron temperature is the most critical point of this paper. We used a fluid approach for the thermal electrons because our low-altitude boundary is at $500 \mathrm{~km}$, where the thermal electron distribution function is very close to a Maxwellian and the thermal conductivity process plays a very important role in the formation of $T_{e}$.

In the work of Tam et al. [1995a], the polarization electric field expression allows for collisionless effects, and it depends on both $T_{e l l}$ and $T_{e \perp}$ [see, e.g., Demars and Schunk, 1992, equation (28)]. In addition, Tam et al. [1995a] used the 16-moment transport equations to describe the flow of both parallel and perpendicular thermal electron energy (through II and $\perp$ heat flows). In the present model, the Spitzer thermal conductivity was used to calculate the electron temperature, which requires a collision-dominated thermal electron population to be valid. However, the thermal electrons are not collision-dominated in the polar wind above altitudes of about 2000-3000 km [e.g., Schunk and Watkins, 1981; Demars and Schunk, 1989]. If the Spitzer conductivity is used for a collisionless plasma, erroneous $T_{e}$ profiles can result. Typically, the Spitzer conductivity tends to keep $T_{e}$ low by rapidly distributing the heat along the geomagnetic field. This may partly explain the difference with the Tam et al. [1995a] calculations, although we think that our treatment should not produce a big difference with a more accurate treatment of the elec- 
tron temperature. In fact, when considering only photoelectron heating of the thermal electrons, the difference in $T_{e}$ between noncollision-dominated and collision-dominated approaches is only $25 \%$ [Gorbachev et al, 1991]. Also, there is another comparison by Olsen and Leer [1996] for the solar wind between a 5-moment and an 8-moment description, and the results show a similar difference in the plasma temperature as was found by Gorbachev et al. [1991].

\section{Conclusion}

In order to answer questions about the maximum expected influence of photoelectrons on the polar wind, we developed a collisionless analytical model of the steady state polar wind with a kinetic description of the ions and photoelectrons and a fluid description of the thermal electrons. This model solves for the electrostatic potential and the electron temperature and describes the quantitative relation between the thermal plasma and the photoelectron parameters. The equations were iteratively solved, using the conditions of quasi-neutrality and currentlessness, and results of this study were presented.

For low-altitude boundary temperatures of $2000 \mathrm{~K}$ for the ions and $2500 \mathrm{~K}$ for the electrons, varying the photoelectron content at the base produced significant changes in the electrostatic potential and thermal electron temperature. For $n_{p 0}=0.01 \%$, the potential drop is $2.87 \mathrm{~V}$ and the temperature reaches $3600 \mathrm{~K}$; for $n_{p 0}=0.10 \%, \varphi_{0}$ is $6.47 \mathrm{~V}$ with a high altitude temperature of $8800 \mathrm{~K}$; and for $n_{p 0}=1.00 \%, \varphi_{0}=7.25 \mathrm{~V}$ and the temperature rises to $17,300 \mathrm{~K}$. Increasing the low-altitude characteristic energies of the electron species increases the potential and electron temperature, while increasing the ion energies decreases the potential. Electron temperatures increase steadily with photoelectron content, always having the same tendency to rise quickly at low altitudes because of the electrostatic coupling and then reach an asymptotic temperature by $2 R_{E}$. The results showed little influence on the location of the upper boundary, changing less than $5 \%$ in $T_{e}$ and less than $1 \%$ in $\varphi$ when the boundary is moved from $5 R_{E}$ to $3 R_{E}$. Inclusion of a source term to represent heating of the thermal electrons through Coulomb collisions with the photoelectrons increases the temperature by less than $1 \%$. In the Ion results, a clear separation occurs between a classical polar wind at low photoelectron concentrations and an $\mathrm{O}^{+}$dominated polar wind for high photoelectron densities.

The model was compared with results from several previous polar wind studies with photoelectrons. A comparison with that of Lemaire [1972] shows good agreement in the features of the results. Lemaire [1972], however, only considered photoelectron concentrations of less than a hundredth of a percent at the base, and thus the results are still within the classical polar wind description of supersonic $\mathrm{H}^{+}$dominance at high altitudes. A comparison with the results of Tam et al. [1995a] shows that the two models show agreement at high altitudes but disagree near the lower boundary. We failed to reproduce the localized enhancement of electron temperature at 500 $1500 \mathrm{~km}$ found by Tam et al. [1995a], but we have very good agreement with the electron temperature profile seen by the Akebono satellite at these altitudes [Abe et al., 1993] for reasonable photoelectron concentrations as predicted by the model of Khazanov and Liemohn [1995].

The critical issue is the electron temperature calculation. This model uses a Spitzer conducitivity description to obtain $T_{e}$, which theoretically is only valid for the thermal electrons in a collision-dominated region. This would only be true at altitudes less that $3000 \mathrm{~km}$ [Demars and Schunk, 1989]. However, it was shown by Gorbachev et al. [1991] and Olsen and Leer [1996] that this assumption should not be greatly different from a more complicated description, and the model shows good agreement with the polar wind measurements of Abe et al. [1993], which indirectly demonstrates the validity of this assumption.

Acknowledgments. This work was supported at MSFC by the NASA Office of Space Science under UPN 432-20-00, at the University of Alabama in Huntsville by NSF grant ATM-9523699, and at the University of Michigan under NASA GSRP grant NGT-51335. G. V. Khazanov held a National Research Council-Marshall Space Flight Center Senior Research Associateship while this work was performed. The authors thank the referees for their many useful comments.

The Editor thanks J.F. Lemaire and R.W. Schunk for their assistance in evaulating this paper.

\section{References}

Abe, T., B. A. Whalen, A. W. Yau, S. Watanabe, E. Sagawa, and K. I Oyama, Altitude profile of the polar wind velocity and its relationship to ionospheric conditions, Geophys. Res. Lett., 20, 2825, 1993.

Axford, W. I.. The polar wind and the terrestrial helium budget, $J$. Geophys. Res., 73, 6855, 1968.

Banks, P.M., and T. E. Holzer, The polar wind, J. Geophys. Res., 73 , 6846,1968

Banks, P.M., and G. Kockarts, Aeronomy, Academic, San Diego, CA, 1973.

Barakat, A. R., and R. W. Schunk, Effect of the hot electrons on the polar wind, J. Geophys. Res., 89, 9771, 1984.

Demars, H. G., and R. W. Schunk, Solutions of bi-Maxwellian transport equations for the polar wind, Planet. Space Sci., 337, 85, 1989.

Demars, H. G., and R. W. Schunk, Semikinetic and generalized transport models of the polar and solar winds, J. Geophys. Res., 97, 1581, 1992.

Ganguli, S. B., The polar wind, Reviews of Geophysics, 34, 311, 1996.

Gorbachev, O. A., Yu.V. Konikov, I. M. Sidorov, and G. V. Khazanov, Allowance for thermal flux variations in the model of ionosphereplasmasphere interactions, Planet. Space Sci., 39, 847, 1991.

Khazanov, G. V., and M. W. Liemohn, Nonsteady state ionosphereplasmasphere coupling of superthermal electrons, J. Geophys. Res., $100,9669,1995$.

Khazanov, G. V., A. F. Nagy, T. I. Gombosi, M. A. Koen, and S. J. Cariglia, Analytic description of the electron temperature behavior in the upper ionosphere and plasmasphere, Geophys. Res. Lett., 19, $1915,1992$.

Khoyloo, A., A. R., Barakat, and R. W. Schunk, On the discontinuity in kinetic solutions of the collisionless polar wind, Geophys. Res. Lett., $18,1837,1991$

Lemaire, J., Effect of escaping photoelectrons in a polar exospheric model, Space Res., 12, 1413, 1972.

Lemaire, J., and M. Scherer, Model of the polar ion-exosphere, Planet Space Sci., 18, 103, 1970.

Lemaire, J., and M. Scherer, Simple model for an ion-exosphere in an open magnetic field, Phys. Fluids, 14, 1683, 1971

Lemaire, J., and $M$. Scherer, Ion-exosphere with asymmetric velocity distribution, Phys. Fluids, 15, 760, 1972.

Miller, R. H., and G. V. Khazanov, A self-consistent electrostatic potential due to trapped plasma in the magnetosphere, Geophys. Res. Lett., 20, 1331, 1993.

Olsen, E. L., and E. Leer, An eight moment solar wind model, in Solar Wind Eight, AIP Conference Proceedings, vol. 382, edited by D. Winterhalter, J. T. Gosling, S. R. Habbal, W. S. Kurth, and M. Neugebauer, p. 157, AIP Press, Woodbury, NY, 1996.

Pollock, C. J., C. R. Chappell, J. L. Horwitz, and J. D. Winningham, Two-spacecraft charged particle observations interpreted in terms of electrostatic potential drops along polar cap field lines, in Modeling Magnetospheric Plasma Processes, Geophys. Monogr. Ser., vol. 62 . edited by G. R. Wilson, p. 111, AGU, Washington, D. C., 1991.

Press, W. H., S. A. Teukolsky, W. T. Vetterling, and B. P. Flannery, Numerical Recipes in FORTRAN, 2nd ed., p. 352, Cambridge Univ. Press, New York, 1992. 
Schunk, R. W., An updated theory of the polar wind, Adv. Space Res., 6(3), 79, 1986.

Schunk, R. W., Polar wind tutorial, in Physics of Space Plasmas (1988), SPI Conf. Proc. Reprint Ser., edited by T. Chang, G. B. Crew, and J. R. Jasperse, Scientific, Cambridge, Mass., 81, 1988a.

Schunk, R. W., The polar wind, in Modeling Magnetospheric Plasma, Geophys. Monogr. Ser., vol. 44, AGU, Washington, D. C., 219, 1988b.

Schunk, R. W., and D. S. Watkins, Electron temperature anisotropy in the polar wind, J. Geophys. Res., 86, 91, 1981.

Tam, S. W. Y., F. J. Yasseen, T. Chang, and S. B. Ganguli, Self-consistent kinetic photoelectron effects on the polar wind, Geophys. Res. Lett., 22, 2107, 1995a.

Tam, S. W. Y., F. J. Yasseen, T. Chang, S. B. Ganguli, and J. M. Retterer, Anisotropic kinetic effects on photoelectrons on polar wind transport, in Cross-Scale Coupling in Space Plasmas, Geophys. Monogr. Ser., vol. 93, edited by J. L. Horwitz, N. Singh, and J. L. Burch, p. 133, AGU, Washington, D. C., 1995b.
Whipple, E. C., Jr., The signature of parallel electric fields in a collisionless plasma, J. Geophys. Res., 82, 1525, 1977.

Wilson, G. R., J. L. Horwitz, D. G. Brown, and G. V. Khazanov, The effect of photoelectrons on plasma outflow at high latitudes, Eos Trans., 77(17 suppl.), 237, 1996.

Winningham, J. D., and C. Gurgiolo, DE-2 photoelectron measurements consistent with a large scale parallel electric field over the polar cap, Geophys. Res. Lett., 9, 977, 1982.

Winningham, J. D., and W. J. Heikkila, Polar cap auroral electron fluxes observed with ISIS I, J. Geophys. Res., 79, 949, 1974.

G.V. Khazanov, M. W. Liemohn, and T. E. Moore, Space Sciences Laboratory, NASA Marshall Space Flight Center, Code ES-83, Huntsville, AL 35812. (email: khazagv@ssl.msfc.nasa.gov)

(Received July 29, 1996; revised September 30, 1996; accepted October 31, 1996.) 
